\title{
Anforderungs- und Bestellprozesse schrittweise digitalisiert
}

Wie können Anwender zugunsten ihrer Kernaufgaben entlastet, die Prozesse für alle Beteiligten effizienter gestaltet und vernetzt werden? Diese Fragen stehen im Mittelpunkt der kontinuierlichen Entwicklung hin zur digitalen Beschaffung an der Uniklinik Köln.

Die Uniklinik Köln steht täglich vor neuen logistischen Herausforderungen: Der innerstädtische Campus umfasst mehr als 100 Gebäude auf rund 220000 Quadratmetern - zahlreiche Bautätigkeiten und Umzüge sorgen für steten Wandel. Mit 1573 Planbetten versorgt der Maximalversorger jährlich über 62000 stationäre Patienten, hinzu kommen noch rund 300000 ambulante Patienten. Mit mehr als 10400 Mitarbeitern aus über 100 Nationen ist die Uniklinik Köln einer der größten Arbeitgeber der Region. 31 von ihnen arbeiten in der Zentralen Beschaffung, die Teil des Geschäftsbereichs Beschaffung und Wirtschaftsbetriebe ist. Aufgeteilt in die drei Teams strategisches und operatives Beschaffungsmanagement sowie Investitionsmanagement verantworten sie den kompletten Einkauf. Die Krankenhausapotheke sowie Bau und Technik arbeiten eigenständig.

Vom Papier zur digitalen Anforderung Die kontinuierlich steigende Zahl der Bestellanforderungen machte die Einführung eines digitalen Anforderungssystems an der Uniklinik Köln notwendig, da ihre Flut manuell nicht mehr zu bewältigen war. Die immer stärkere Vernetzung einzelner Abteilungen und voneinander abhängige Prozesse kamen als Herausforderung hinzu. Bereits 2012 etablierte die Zentrale Beschaffung daher das Supplier Relationship Management (SRM) als flächendeckendes digitales Anforderungssystem und baute es stetig aus.

Die Anzahl der Bestellpositionen stieg von knapp über 100000 im Jahr 2010

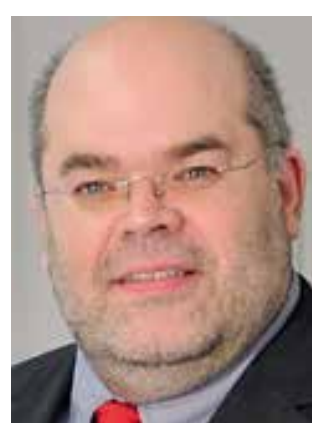

Foto: Michael Wodak/ MedizinFotoKöln

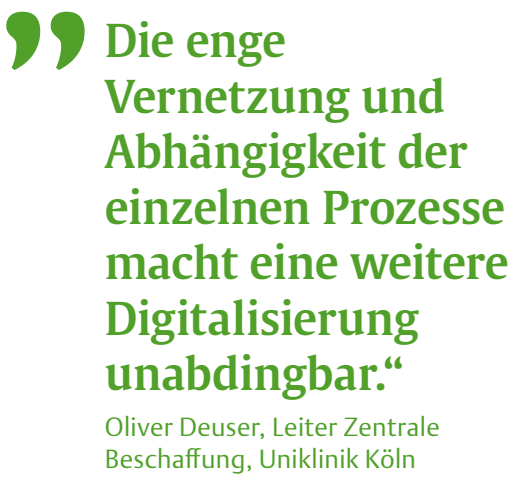

auf mehr als das Doppelte im Jahr 2017. Das SRM entwickelte sich vom einfachen zum hochkomplexen System, das durch permanente Optimierung und immer neue Funktionen für die rund 3000 Anwender in den verschiedenen Bereichen der Uniklinik Köln heute nicht mehr wegzudenken ist. Das Besondere: Die überwiegenden Änderungen und Verbesserungen kommen von den Beschäftigten der Zentralen Beschaffung, die „ihr“ System kontinuierlich verbessern. Standard-Artikel, zu denen unter anderem Lagerartikel zählen, werden als Stammdaten im SAP abgebildet. Um

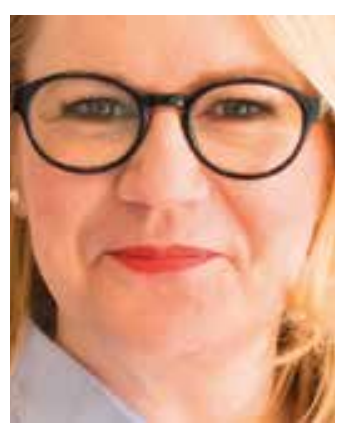

Foto: Kerstin Groß

29 Die Anforderungen an die Anwender werden immer komplexer, daher sollte der Bestellprozess einfach und komfortabel gestaltet sein.“

Kerstin Groß, Zentrale Beschaffung, Uniklinik Köln

zügig große Sortimente beispielsweise aus dem Laborbereich darzustellen und zu listen, sind zusätzlich komplette Preislisten verfügbar.

Einfach und übersichtlich dank Webshops

In einem weiteren Schritt wurden Webshops über eine OCI-Schnittstelle an das SRM-Bestellsystem angebunden. Ist der Anwender im SRM angemeldet und besucht beispielsweise einen Webshop für Laborbedarf, sieht er dort alle Daten, die im Internet verfügbar sind. Zusätzlich sieht er auch die verhandelten Preise der 

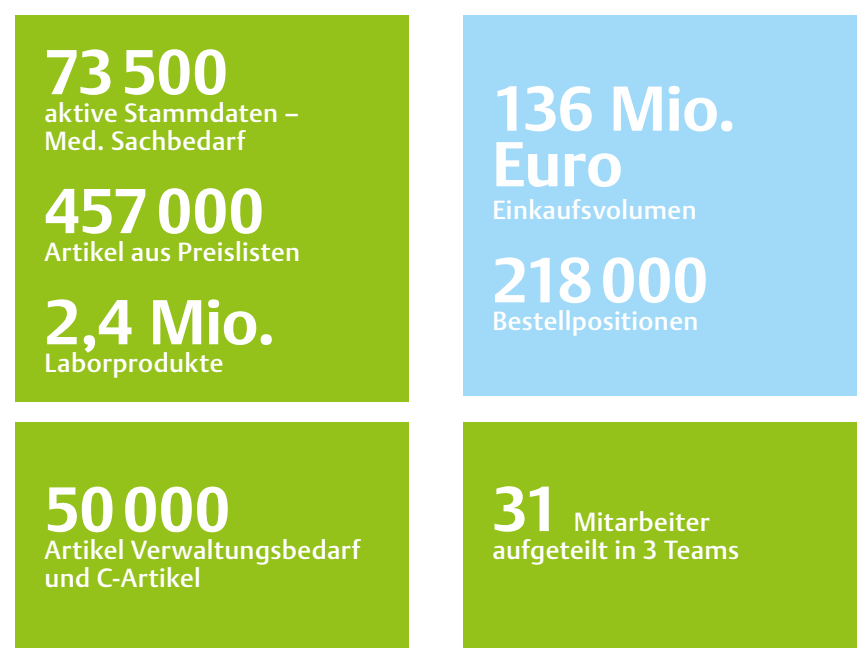

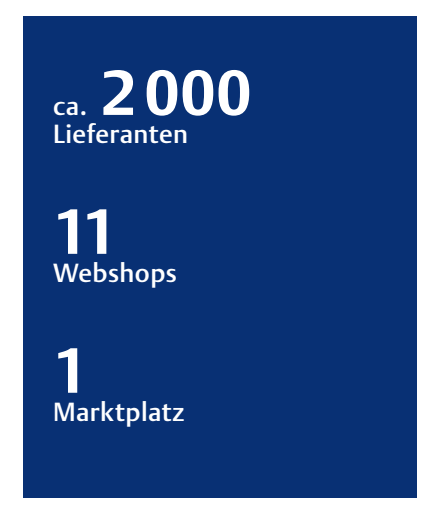

Quelle: Zentrale Beschaffung, Universitätsklinikum Köln Die Uniklinik Köln gehört zu den größten Maximalversorgern in Deutschland.
Zentralen Beschaffung. Er kann also direkt aus dem Webshop Produkte zu realen Konditionen anfordern - ohne Anfrage bei der Beschaffung oder Angebote beim Lieferanten einholen zu müssen.

Da die Webshops mehr Informationen bieten und aussagekräftiger sind als Stammdaten oder eine einfache SAPListung, wurden im Rahmen eines gesonderten Projekts weitere Anbindungen umgesetzt. So kamen Shops für die Bereiche Printmedien, Büromöbel und IT-Bedarf hinzu. Ziel war auch, die Warengruppen sowie den Wirtschafts- und Verwaltungsbedarf zu strukturieren, denn anders als im Medical- und Laborbereich gab es keine definierten Standards. Da dieses Sortiment auch viele typische CArtikel beinhaltet, war schnell klar, dass eine Lösung notwendig war, die einerseits eine große Sortimentsvielfalt bieten und anderseits eine zügige automatisierte Bestellung ermöglichen sollte.

Im Bereich Verwaltungsbedarf wurde über einen Büro-Artikel-Webshop ein durchdachtes Kernsortiment zur Verfügung gestellt, welches heute circa 2000 Artikel umfasst. Später folgte die Anbindung eines eigenen Toner-Webshops. Mittels des ebenfalls realisierten Stempel-Konfigurators kann der Anwender gängige Stempel selbst im Corporate Design erstellen und automatisch anfordern. Die früher aufwändige Anforderung inklusive Freigabe des Drucktextes entfiel. Eine weitere Verbesserung war die Möglichkeit der Anwender, in jedem Shop eine individuelle Favoritenliste anlegen zu können. Hier können alle regelmäßig zu bestellenden Artikel hinterlegt werden, ohne dass der Anwender bei jedem Bestellvorgang erneut im kompletten Sortiment suchen muss.

\section{Anwenderfreundlicher Bestellprozess} Eine wesentliche Herausforderung für das Team der Zentralen Beschaffung war die Akzeptanz der Anwender. Ein einfach gestalteter Bestellprozess sollte der sehr heterogenen Anwenderstruktur mit Ärzten und Pflege in der Krankenversorgung, aber auch den Anforderungen der Beschäftigten in Lehre und Forschung gerecht werden. Ziel war es, dass die Anwender so wenig Zeit wie möglich für die Anforderung von Materialien und Dienstleistungen benötigen, um sich ihren Kernaufgaben in Krankenversorgung, Forschung und Lehre zu widmen.

Das Change-Management war dabei ein wichtiger Aspekt, daher wurden bei allen Prozessen und Neuerungen stets auch die Anwender einbezogen. Weiterentwicklungen und neue Abläufe mussten zunächst ihre Testung bestehen und wurden erst nach Freigabe eingeführt.
Ein Vorgehen das für positives Feedback, Rückmeldungen bei Schwierigkeiten und Verbesserungsvorschläge von den Anwendern sorgte.

Als vorerst letzte Stufe wurden weitere Bereiche in das System eingebunden, um Anforderungen prüfen zu können. So laufen beispielsweise Anforderungen aus dem Bereich Medizintechnik oder IT zur Prüfung automatisch in die entsprechenden Fachbereiche. Erst nach Freigabe durch die Fachabteilung, kommt der Warenkorb in die Zentrale Beschaffung. Damit ist die Einbindung der Fachbereiche sichergestellt. Das vorher zeitaufwändige und fehleranfällige Hin-und-her-Senden von Anforderungen entfällt. Gleichzeitig kann der Anwender jederzeit sehen, wo der Warenkorb liegt und wie der Bearbeitungsstand ist.

\section{Einbindung einer B2B-Plattform für C-Artikel}

Um auch innerhalb der Zentralen Beschaffung Abläufe zu optimieren und neben dem bestehenden SRM-System weitere Tools für eine schnelle und kostenschonende Bearbeitung von Anforderungen zu etablieren, ist ein Marktplatz für C-Artikel geschaffen worden. Aufgrund der bereits bestehenden Zusammenarbeit mit der B2B-Beschaffungsplattform Mercateo wurde diese Zusammenarbeit entsprechend intensiviert. 
Die Vorteile für die Zentrale Beschaffung liegen in der vielfältigen Lieferanten- und Produktauswahl. Bei eingehenden Freitext-Anforderungen, die weder im Standard- noch in einem anderen Sortiment vorhanden sind, entfällt eine aufwendige Recherche. Das Einholen von Vergleichsangeboten ist nicht mehr notwendig, da der Marktplatz in der Regel einen guten Wettbewerb bietet. Ein weiterer Vorteil: Lieferanten müssen nicht mehr angelegt und gepflegt werden, da die Rechnungsstellung immer über Mercateo erfolgt. Ein großer Prozesskosten-Vorteil, gerade bei Produkten, die nur einmal oder sehr unregelmäßig beschafft werden und nicht zum Kernsortiment gehören. Die Neuerungen entlasten nicht nur die Mitarbeiter der Beschaffung, sondern auch die Buchhaltung und die Rechnungsstelle.

Darüber hinaus bietet die B2B-Plattform die Listung eigener Kataloge mit eigenen verhandelten Konditionen an. Herausragend ist die Best-Basket-Funktion: Gegen Ende des Bestellprozesses wird dabei der Warenkorb automatisch optimiert, indem die Kombination aus Preis, Lieferzeit und Versandkosten geprüft und gegebenenfalls korrigiert wird. Insgesamt führt der Markplatz dazu, dass Freitextanforderungen zu C-Artikeln schneller und günstiger abgewickelt werden können.

\section{Digitalisierung geht auch 2019 weiter} Trotz früher Etablierung eines digitalen Anforderungssystems und kontinuierlicher Weiterentwicklung ist klar, dass es sich immer noch um die ersten Schritte hin zum digitalen Einkauf handelt. Ein neues Projekt steht bereits in den Startlöchern und beinhaltet die Einführung einer übergreifenden BestellPlattform. Hiermit erreicht das bestehende System eine neue Stufe und die Zentrale Beschaffung der Uniklinik Köln einen weiteren wichtigen Meilenstein.
Hauptmerkmal der neuen Funktion ist die Cross-Katalog-Suche. Damit hat der Anwender die Möglichkeit, mit einer Suche alle angebundenen Stammdaten, Preislisten, Webshops und vieles mehr auf der Ebene einzelner Artikel direkt zu vergleichen. Bereits vor der Anforderung bei der Zentralen Beschaffung,

\section{Ohne \\ Digitalisierung der Bestellanfor- derungen wäre die Arbeit nicht mehr zu bewältigen.“ Tanja Deuser, Leiterin Operativer Einkauf, Uniklinik Köln}

sieht der Anwender so die verschiedenen Produkte, Preise und Lieferzeiten und kann das für ihn beste Angebot auswählen. Gerade im Forschungsbereich ein enorm wichtiger Aspekt, da hier eine große Zahl vergleichbarer Produkte in den Webshops zu finden ist und der aufwändige Lieferantenvergleich entfällt. Mit der ebenfalls schon heute umgesetzten Echtzeit-Budgetprüfung, kann er prüfen, ob das ausgewählte Produkt ins vorhandene Budget passt und es mit einem Klick anfordern.

Mit den neuen Funktionen der BestellPlattform wäre auch eine Abwicklung weiterer Anforderungen denkbar. So könnten beispielweise über gesonderte Formulare Dienstleistungen der Medizintechnik in Anspruch genommen oder Aufträge in die Hausdruckerei gesendet werden. Ein weiteres Beispiel wäre die Einbindung des Caterings. So wäre die Bestellung der Dienstleistung des Tochterunternehmens digital über ein bestehendes System möglich.

\section{Ein System - viele Möglichkeiten}

Der Zentralen Beschaffung der Uniklinik Köln ist es wichtig, den Bestellprozess stetig zu verbessern und den Anwendern ein gut funktionierendes, durchdachtes System anzubieten. Wichtig ist und war, dass das System nicht nur für die einfache Anforderung genutzt werden kann, sondern auch für andere Dienstleistungen der Uniklinik und der Tochterunternehmen, und der Anwender letztlich nur ein System für alle Anforderungen benötigt. Nur so können zeitaufwändige Freitextanforderungen, Schulungen, Pflege und Support verschiedener IT-Systeme und Schnittstellenproblematiken vermieden werden.

Auch wenn der Aufwand stellenweise sehr groß ist und viel Überzeugungsarbeit kostet, sind die Experten von den vielen Vorteilen überzeugt und wollen die bestehenden Systeme ausbauen, stetig prüfen und verbessern. Nur so kann die Beschaffung dazu beitragen den Verwaltungsaufwand für die Anwender in Patientenversorgung, Forschung und Lehre so gering wie möglich zu halten, damit sie ihrer Hauptaufgabe mehr Zeit widmen können.

Kerstin Groß, Geschäftsbereich Beschaffung und Wirtschaftsbetriebe, Zentrale Beschaffung, Uniklink Köln.

Oliver Deuser, Leiter Zentrale Beschaffung, Uniklinik Köln 